\title{
FURTHER EVIDENCE OF A RELATIVE LACK OF C-21 HY- DROXYLATION IN CONGENITAL ADRENAL HYPERPLASIA *
}

\author{
By ANDREW G. FRANTZ, $\dagger$ DONALD A. HOLUB AND JOSEPH W. JAILER \\ (From the Departments of Medicine and Obstetrics and Gynecology, College of Physicians and \\ Surgeons, Columbia University, and the Presbyterian Hospital, New York, N. Y.)
}

(Submitted for publication December 16, 1959; accepted February 19, 1960)

Although it is generally accepted that in congenital adrenal hyperplasia with virilism there is a relative lack of certain hydroxylating enzymes within the adrenal cortex ( $\mathrm{C}-21$ and $\mathrm{C}-11)$, the ultimate proof is not as yet at hand. The evidence supporting this hypothesis is based on several types of data. A characteristically abnormal urinary steroidal pattern has been found in patients with congenital adrenal hyperplasia $(1,2)$. Since these patients apparently metabolize exogenously administered steroids in a normal fashion (3), the basic defects in this disease must reside within the adrenal cortex. The characteristic urinary steroidal pattern has been approximated by the administration of the cortisol precursors, 17hydroxyprogesterone and 21-desoxyhydrocortisone, to hypoadrenal patients (1). Seventeenhydroxyprogesterone, rather than cortisol, has been found to be the main steroidal constituent of extracts from adrenal glands of patients with congenital adrenal hyperplasia (4). A submaximal rise in the urinary and plasma 17-hydroxycorticosteroids has been observed as a result of $\mathrm{ACTH}$ administration to these patients $(2,5,6)$, indicating a relative inefficiency in the secretion of cortisol.

All these data would support the hypothesis that cortisol is synthesized with difficulty and that certain cortisol precursors accumulate in the adrenal gland and escape into the circulation. However, Cope has recently demonstrated in one patient with this condition an increased cortisol secretion rate, tending to cast some doubt on the above described pathogenesis of congenital adrenal hyperplasia (7).

* Aided by grants from the United States Public Health Service A 195 (C7) and A 2208(C), and the Upjohn Company.

+ Trainee, Institute of Arthritis and Metabolism, National Institutes of Health; now on active duty, U. S. Navy.
An amphenone derivative, 2-methyl-1,2-bis-(3pyridyl)-propanone (SU-4885), has become available which, when administered in the proper dosage to human subjects and experimental animals, selectively inhibits $11 \beta$-hydroxylation $(8,9)$. Under these circumstances, 11-desoxyhydrocortisone (Compound $\mathrm{S}$ ) is produced in normal individuals in place of cortisol. In an attempt to shed additional light on the underlying pathogenesis of this disease, SU-4885 was administered to patients with congenital adrenal hyperplasia.

\section{METHODS AND MATERIALS}

After adequate control periods of daily urine collections, $750 \mathrm{mg}$ of SU-4885 was administered orally every 4 hours for 6 doses, and urine collections were continued for at least one day after the cessation of the drug administration. Three normal individuals (A.F., M.N. and D.M.) were used as controls, whereas three patients with documented congenital adrenal hyperplasia, not receiving steroid therapy (E.B., D.P. and M.G.), were given SU-4885 under identical circumstances.

For the study of C-21 urinary metabolites, aliquots of the 24-hour urine specimens were hydrolyzed with $\beta$-glucuronidase, $^{1} 750 \mathrm{U}$ per $\mathrm{ml}$ for 48 hours at $\mathrm{pH} 5.0$, and extracted twice with equal volumes of ethyl acetate. After being washed with small volumes of $1 \mathrm{~N} \mathrm{NaOH}$ and distilled water, the extracts were dried, and portions representing 1 to 4 per cent of the day's output were applied to 1 inch wide strips of methanol-washed Whatman no. 3MM chromatography paper. Adjacent to each 1 inch strip a 0.5 inch marker strip was run, to which had been applied the same extract in one-half the amount. A parallel 1 inch strip carrying $25 \mu \mathrm{g}$ amounts of the appropriate standards was employed in each chromatographic run. After being placed in a Bush $B_{5}$ tank (10), the strips were equilibrated for 3 hours, and the solvent was then allowed to run a distance of $40 \mathrm{~cm}$ during about 2.5 hours. Equilibration and running were both done at room temperature.

After the paper had been dried in room air for a few minutes, steroids were located on the standard and marker strips by treatment with blue tetrazolium (BT) reagent. Parallel areas of the untreated 1 inch strip, rep-

${ }^{1}$ Ketodase, Warner-Chilcott. 
resenting tetrahydrocortisol (THF), allotetrahydrocortisol (allo-THF) and tetrahydrocortisone (THE) together near the upper end, and tetrahydro-11-desoxycortisol (THS), ${ }^{2}$ further down, were then cut out. These were eluted chromatographically on a modified Haines device (11), using three successive $7 \mathrm{ml}$ washings of methanol: ethyl acetate, $1: 1$, from alternate corners of the strip; the eluates were dried under air at $40^{\circ} \mathrm{C}$ in a test tube.

From most urinary extracts, THS emerged on chromatography as a rather diffuse and faint band at normal concentrations. Therefore, further purification of this steroid was carried out in all cases by rechromatography of the appropriate eluate in a Bush $B_{1}$ system (10), in a manner similar to that described above, except that the paper was usually over-run for 10 to 16 hours. Under these conditions THS always separated out as a sharply defined 1 to $2 \mathrm{~cm}$ wide band from a number of other associated steroids, both positive and negative to BT, as well as various nonsteroidal contaminants, including metabolites of SU-4885. The THS was then eluted as described and taken to dryness.

Quantitation of the steroids was carried out by means of the Silber-Porter reaction (12). The dried eluates were dissolved in varying amounts of absolute ethanol and aliquots were taken so that concentrations (estimated visually from the intensity of the BT spots) would result in similar final readings on the Beckman model DU spectrophotometer. To each ethanolic solution was then added twice its volume of a freshly prepared solution of $32.5 \mathrm{mg}$ recrystallized phenylhydrazine in $50 \mathrm{ml}$ of $11.2 \mathrm{M} \mathrm{H}_{2} \mathrm{SO}_{4}$. In addition, a blank was run for each eluate, consisting of identical amounts of the same ethanolic solutions treated with double volumes of $11.2 \mathrm{M} \mathrm{H}_{2} \mathrm{SO}_{4}$ without phenylhydrazine. The samples and their blanks, together with two reagent blanks containing the ethanol-sulfuric acid mixture with and without phenylhydrazine, were allowed to stand overnight at room temperature. They were then read on the spectrophotometer at 370,410 and $450 \mathrm{~m} \mu$. From the readings obtained with the phenylhydrazine mixture, the corresponding readings obtained with $\mathrm{H}_{2} \mathrm{SO}_{4}$ alone were subtracted at all three wave lengths and the final quantities were calculated by use of the Allen correction (13) in comparison with known standards of THE and THS, both of which were run through the final color reaction.

Experience with this method has shown consistent recovery figures averaging 90 per cent, when one chromatographic step was used, and 80 per cent, when there were two chromatographic steps; these corrections were therefore applied to the final figures. Excellent approximation of the pure Silber-Porter spectrophotometric curve was noted in every case after subtraction of the sulfuric acid blank, and in most cases before sub-

2 THF , $3 \alpha, 11 \beta, 17 \alpha, 21$ - tetrahydroxy - pregnan-20-one ; allo-THF, $3 \alpha, 11 \beta, 17 \alpha, 21$ - tetrohydroxy - allopregnan-20one; THE, $3 \alpha, 17 \alpha, 21$-trihydroxy-pregnane-11,20-dione ; THS. $3 \alpha, 17 \alpha, 21$-trihydroxy-pregnan-20-one. traction as well, so that the use of this blank made little difference to the final Allen-corrected value.

Identification of the measured group of cortisol metabolites was not carried out beyond establishing the identity of Rf values with those of standards, plus appropriate reactions to UV light, BT, and Silber-Porter reagents; the last of these, as used here with individual blanks and readings at three wave lengths, constitute a reasonably good test of specificity for the presence of a 21-hydroxyl group in a dihydroxyacetone configuration. Criteria for the identification of THS, in addition to those mentioned above, included in all cases the identity of $\mathrm{Rf}$ in a second chromatographic system, plus in most cases the preparation and chromatography of a bismuthate oxidation derivative which was Zimmermann positive and had a mobility identical with that of etiocholanolone. In addition, from one 24-hour urine after SU-4885 administration, following multiple paper chromatography, several milligrams of a substance were crystallized which had an infrared spectrum identical with that of authentic THS, and a melting point of 184 to $186^{\circ} \mathrm{C}$, which agrees closely with that of 184 to $185^{\circ} \mathrm{C}$ reported for THS by Eberlein and Bongiovanni (14).

It should be noted that values for the THF, allo-THF, THE group are expressed in terms of a THE standard, and those for THS in terms of a THS standard. Employing the Silber-Porter reaction as described, it was found that THS has a chromogenicity which is 80 per cent that of THE.

The 17-ketosteroids and total ketogenic steroids were determined by the method of Norymberski, Stubbs and West (15). Pregnanetriol ${ }^{3}$ was analyzed by the Bongiovanni and Eberlein technique (16), evidence for the specificity of which has been discussed by the authors.

\section{RESULTS}

Normal subjects. The administration of SU4885 to normal subjects resulted in a marked increase in the urinary ketogenic steroids, reaching maximal levels in the 24 hours following the administration of the drug. Consequently, urines collected during this period were chosen for steroidal analysis (Table I).

Chromatographic analysis of the cortisol metabolites revealed control values similar to those reported by others $(17,18)$. The control values of THS, however, are somewhat higher than have been noted previously (19). After SU-4885 administration the cortisol metabolites were considerably reduced, and THS increased to levels as high as $20 \mathrm{mg}$ per day. Jenkins and co-workers (20) have noted a similar rise in urinary THS when SU-4885 was given to normal subjects. A modest increase in urinary pregnanetriol

\footnotetext{
${ }^{3}$ Pregnane-3 $\alpha, 17 \alpha, 20 \alpha$-triol and related isomers.
} 
TABLE I

C-21 urinary steroids before and after SU-4885 administration in patients with congenital adrenal hyperplasia and in normal controls

\begin{tabular}{|c|c|c|c|c|c|c|c|c|c|}
\hline & & \multicolumn{2}{|c|}{$\begin{array}{c}\text { Total 17-ketogenic } \\
\text { steroids }\end{array}$} & \multicolumn{2}{|c|}{$\begin{array}{l}\text { Tetrahydro } F \text {, } \\
\text { allotetrahydro } \mathrm{F} \text {, } \\
\text { and tetrahydro } \mathrm{E}\end{array}$} & \multicolumn{2}{|c|}{ Tetrahydro S } & \multicolumn{2}{|c|}{ Pregnanetriol } \\
\hline & & Before & After & Before & After & Before & After & Before & After \\
\hline & & \multicolumn{2}{|c|}{$m g / d a y$} & \multicolumn{2}{|c|}{$m g / d a y$} & \multicolumn{2}{|c|}{$m g / d a y$} & \multicolumn{2}{|c|}{$m g / d a y$} \\
\hline Normal controls & $\begin{array}{l}\text { D. M. } \% \\
\text { M. N. } \\
\text { A. F. } \sigma^{7}\end{array}$ & $\begin{array}{l}10.5 \\
10.7 \\
13.3\end{array}$ & $\begin{array}{l}24 \\
49 \\
27\end{array}$ & $\begin{array}{l}1.7 \\
5.4 \\
3.3\end{array}$ & $\begin{array}{l}0.70 \\
2.8 \\
2.6\end{array}$ & $\begin{array}{l}0.071 \\
0.077 \\
0.14\end{array}$ & $\begin{array}{l}10.3 \\
20.0 \\
14.0\end{array}$ & $\begin{array}{l}1.9 \\
3.9 \\
2.0\end{array}$ & $\begin{array}{r}5.8 \\
14.2 \\
5.8\end{array}$ \\
\hline $\begin{array}{l}\text { Congenital adrenal } \\
\text { hyperplasia patients }\end{array}$ & 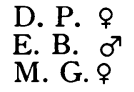 & $\begin{array}{l}46 \\
21 \\
61\end{array}$ & $\begin{array}{l}240 \\
122 \\
300\end{array}$ & $\begin{array}{l}1.3 \\
2.2 \\
2.2\end{array}$ & $\begin{array}{l}1.0 \\
0.52 \\
1.4\end{array}$ & $\begin{array}{c}* \\
* \\
0.12\end{array}$ & $\begin{array}{l}1.8 \\
5.5 \\
5.6\end{array}$ & $\begin{array}{l}54 \\
15 \\
45\end{array}$ & $\begin{array}{r}220 \\
99 \\
250\end{array}$ \\
\hline
\end{tabular}

${ }^{*}$ Because of the very small amounts of THS judged to be present in these specimens ( $<40 \mu g$ per day), rechromatography and quantitation were not carried out.

values was also noted after SU-4885 treatment (Table I).

The urinary excretion of 17 -ketosteroids rose variably following the administration of SU-4885 (Table II).

Patients with congenital adrenal hyperplasia. During the control period, decreased to normal amounts of THE, THF and allo-THF could be detected in the urine of all three patients with congenital adrenal hyperplasia. On the other hand, the pregnanetriol values were consistently elevated (Table I), accounting in large part for the elevated ketogenic steroids manifested by these patients during the control period.

Administration of SU-4885 to these three patients resulted in a considerably greater increase in the total urinary ketogenic steroids than was found in normal subjects. Although an increase in THS occurred in this group, it was of much smaller magnitude than that observed in the normal group and constituted only a relatively minute share of the total ketogenic steroids. The bulk of the ketogenic steroids was found in the

TABLE II

Total urinary 17-ketosteroids before and after $S U-4885^{*}$

\begin{tabular}{|c|c|c|c|}
\hline & Subject & Before & After \\
\hline Normal controls & $\begin{array}{l}\text { A. F. } \sigma^{7} \\
\text { D. M. } \\
\text { M. N. }\end{array}$ & $\begin{array}{r}7.0 \\
2.8 \\
13.7\end{array}$ & $\begin{array}{r}9.4 \\
7.3 \\
26.5\end{array}$ \\
\hline $\begin{array}{l}\text { Congenital adrenal } \\
\text { hyperplasia patients }\end{array}$ & 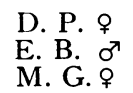 & $\begin{array}{l}21.4 \\
32 \\
45\end{array}$ & $\begin{array}{l}48.6 \\
50 \\
91\end{array}$ \\
\hline
\end{tabular}

* Milligram per 24 hours. pregnanetriol fraction. Diminished excretion of the metabolites of cortisol was also noted, similar to that seen in the normal controls. The urinary 17 -ketosteroids rose in all patients following SU-4885 administration (Table II).

\section{DISCUSSION}

Liddle and associates (8) and Jenkins and associates (9) have previously shown that the amphenone derivative, SU-4885, can cause a specific inhibition of $11 \beta$-hydroxylation within the adrenal cortex, resulting in the secretion of Compound $\mathrm{S}$ instead of the usual secretory product, cortisol. Since Compound S is only a weak inhibitor of the anterior pituitary, the latter secretes increased amounts of $\mathrm{ACTH}$, stimulating the adrenal cortex to synthesize quantities of Compound $\mathrm{S}$ and other 11-desoxycorticosteroids in considerable excess of the normal secretion of cortisol. This is reflected in the finding of elevated urinary ketogenic steroids, of which the major components in normal subjects are metabolites of Compound S instead of the usually occurring metabolites of cortisol.

Although the urinary ketogenic steroids rise in both groups of individuals after the administration of SU-4885, the component urinary metabolites are quite different in congenital adrenal hyperplasia from those found in normal subjects. In the latter group, THS is present in large quantities; in the former group the main urinary metabolite is pregnanetriol, a degradation product of $17 \alpha$-hydroxyprogesterone.

It had previously been postulated that in con- 
genital adrenal hyperplasia the two main adrenal C-21 secretory products were 17-hydroxyprogesterone and 21-desoxyhydrocortisone (1). With inhibition of $11 \beta$-hydroxylation induced by SU4885 , the production of the latter steroid is reduced, as well as that of cortisol, and the major C-21 steroid secreted is 17-hydroxyprogesterone.

Thus, when $11 \beta$-hydroxylation is inhibited chemically, the normal individual secretes Compound $\mathrm{S}$, whereas the patient with congenital adrenal virilism secretes principally 17 -hydroxyprogesterone. These data confirm the hypothesis that at least one of the enzymatic defects in congenital adrenal hyperplasia is at the C-21 hydroxylating position.

It is of interest the SU-4885 causes a defect in the normal adrenal biosynthetic pathways similar to that postulated by Eberlein and Bongiovanni as occurring in the hypertensive form of adrenal virilism, namely, a relative lack of $11 \beta$-hydroxylation (14).

The finding, in patients with congenital adrenal hyperplasia, of tetrahydro derivatives of cortisol whose amounts overlap the normal range has been reported previously $(21,22)$ and does not exclude a relative defect in 21 -hydroxylation as the basic mechanism of the disease.

The present studies demonstrate in all patients an ample pituitary reserve of $\mathrm{ACTH}$; following its release, adrenal reserve capacity to form cortisol precursors is exhibited to a marked degree, as measured by the rise in the already elevated pregnanetriol values. Thus long continued pituitary-adrenal overactivity apparently does not diminish, and may conceivably even enhance, the responsiveness of this system to additional stimulation. The limiting factor in the congenital adrenal hyperplasia group lies in the 21-hydroxylation system, where a definitely subnormal response to SU-4885 administration occurs.

\section{SUMMARY}

1. Three patients with congenital adrenal hyperplasia and three normal subjects were treated with 2-methyl-1,2-bis-(3-pyridyl)-propanone (SU-4885), a synthetic inhibitor of $11 \beta$-hydroxylation in the adrenal cortex. By causing the formation of 11-desoxysteroids which lack pituitaryinhibiting properties, SU-4885 indirectly stimu- lated pituitary release of $\mathrm{ACTH}$, and therefore all subjects demonstrated increased excretion of urinary ketogenic steroids and 17-ketosteroids.

2. Individual urinary $\mathrm{C}-21$ steroidal metabolites were separated chromatographically and quantitated, before and after SU-4885 administration. Cortisol metabolites decreased in the normal subjects in response to SU-4885 and were replaced by large amounts of tetrahydro S, a metabolite of 11desoxyhydrocortisone. In the adrenal hyperplasia group, initially low levels of cortisol metabolites declined still further after SU-4885; tetrahydro $S$ appeared, but in much smaller amounts than in the normal subjects. Pregnanetriol, however, rose strikingly after SU-4885 from initially elevated values in the hyperplasia group.

3. In addition to demonstrating a considerable pituitary-adrenal reserve, these data are interpreted as providing further evidence of a relative defect of 21 hydroxylation in congenital adrenal hyperplasia.

\section{ACKNOWLEDGMENTS}

The authors are indebted to Dr. C. H. Sullivan of Ciba Pharmaceutical Products, Inc., for generously supplying the SU-4885 used in these studies. Miss Elsie Ewen and Miss Margaret Welch supplied invaluable technical assistance.

\section{REFERENCES}

1. Jailer, J. W., Gold, J. J., Vande Wiele, R., and Lieberman, S. $17 \alpha$-Hydroxyprogesterone and 21 desoxyhydrocortisone; their metabolism and possible role in congenital adrenal virilism. J. clin. Invest. 1955, 34, 1639.

2. Bongiovanni, A. M., Eberlein, W. R., and Cara, J. Studies on the metabolism of adrenal steroids in the adrenogenital syndrome. J. clin. Endocr. 1954, 14, 409.

3. Fukushima, D. K., and Gallagher, T. F. Absence of 21-dehydroxylation in congenital adrenal hyperplasia. J. clin. Endocr. 1958, 18, 694.

4. Bongiovanni, A. M. In vitro hydroxylation of steroids by whole adrenal homogenates of beef, normal man, and patients with the adrenogenital syndrome. J. clin. Invest. 1958, 37, 1342.

5. Kelley, V. C., Ely, R. S., and Raile, R. B. Metabolic studies in patients with congenital adrenal hyperplasia. Effects of cortisone therapy. J. clin. Endocr. 1952, 12, 1140.

6. Christy, N. P., Wallace, E. Z., and Jailer, J. W. The effect of intravenously-administered ACTH on plasma 17,21-dihydroxy-20-ketosteroids in normal individuals and in patients with disorders of the adrenal cortex. J. clin. Invest. 1955, 34, 899. 
7. Cope, C. L. Steroid metabolism in a case of congenital adrenal hyperplasia. Brit. med. J. 1959, 1,815 .

8. Liddle, G. W., Island, D., Lance, E. M., and Harris, A. P. Alterations of adrenal steroid patterns in man resulting from treatment with a chemical inhibitor of $11 \beta$-hydroxylation. J. clin. Endocr. 1958, 18, 906.

9. Jenkins, J. S., Meakin, J. W., Nelson, D. H., and Thorn, G. W. Inhibition of adrenal steroid 11oxygenation in the dog. Science 1958, 128, 478.

10. Bush, I. E. Methods of paper chromatography of steroids applicable to the study of steroids in mammalian blood and tissues. Biochem. J. 1952, 50, 370.

11. Haines, W. J. Studies on the biosynthesis of adrenal cortex hormones. Recent Progr. Hormone Res. 1952, 7, 255.

12. Silber, R. H., and Porter, C. C. The determination of 17, 21-dihydroxy-20-ketosteroids in urine and plasma. J. biol. Chem. 1954, 210, 923.

13. Allen, W. M. A simple method for analyzing complicated absorption curves of use in the colorimetric determination of urinary steroids. J. clin. Endocr. 1950, 10, 71.

14. Eberlein, W. R., and Bongiovanni, A. M. Plasma and urinary corticosteroids in the hypertensive form of congenital adrenal hyperplasia. J. biol. Chem. 1956, 223, 85.

15. Norymberski, J. K., Stubbs, R. D., and West, H. F. Assessment of adrenocortical activity by assay of 17-ketogenic steroids in urine. Lancet 1953, 1, 1276.

16. Bongiovanni, A. M., and Eberlein, W. R. Critical analysis of methods for measurement of pregnane3-alpha, 17-alpha, 20-alpha-triol in human urine. Analyt. Chem. 1958, 30, 388.

17. Bush, I. E., and Willoughby, M. The excretion of allo tetrahydrocortisol in human urine. Biochem. J. 1957, 67, 689.

18. Romanoff, L. P., Rodriguez, R. M., Seelye, J. M., Parent, C., and Pincus, G. Urinary excretion of tetrahydrocortisol, $3 \alpha$-allotetrahydrocortisol and tetrahydrocortisone in young and elderly men and women. J. clin. Endocr. 1958, 18, 1285.

19. Touchstone, J. C., Bulaschenko, H., Richardson, E. M., and Dohan, F. C. The excretion of pregnane-3 $\alpha$-17 $\alpha, 21$-triol-20-one (tetrahydro $S$ ) in normal and pathologic urine. J. clin. Endocr. 1957, $17,250$.

20. Jenkins, J. S., Pothier, L., Reddy, W. J., Nelson, D. H., and Thorn, G. W. Clinical experience with selective inhibition of adrenal function. Brit. med. J. 1959, 1, 398.

21. Bongiovanni, A. M., and Eberlein, W. R. Defective steroidal biogenesis in congenital adrenal hyperplasia. Pediatrics 1958, 21, 661.

22. Jailer, J. W., Ulick, S., and Lieberman, S. Aldosterone and hydrocortisone secretion rates in patients with the salt-losing form of congenital adrenal hyperplasia. Trans. Ass. Amer. Phycns 1959, 72, 149. 